\title{
ON EMBEDDING CIRCLE-BUNDLES IN FOUR-MANIFOLDS
}

\author{
Peter Ozsváth And Zoltán Szabó
}

\begin{abstract}
In this paper, we demonstrate an obstruction to finding certain splittings of four-manifolds along sufficiently twisted circle bundles over Riemann surfaces, arising from Seiberg-Witten theory. These obstructions are used to show a non-splitting result for algebraic surfaces of general type.
\end{abstract}

\section{Introduction}

Let $Y(n, g)$ denote the circle bundle over a genus $g$ surface with Euler number $n$. Our main result in this paper is the following:

Theorem 1.1. If $X$ is a complex surface of general type, and $Y(n, g)$ is circlebundle over a Riemann surface of genus $g$, whose Euler number $n$ satisfies $|n| \geq$ $2 g-1$, then $X$ admits no splittings along an embedded copy of $Y=Y(n, g)$ of the form $X=X_{1} \#_{Y} X_{2}$ with $b_{2}^{+}\left(X_{1}\right), b_{2}^{+}\left(X_{2}\right)>0$.

In the above theorem, the quantity $b_{2}^{+}(Z)$ of a four-manifold $Z$ with boundary denotes the maximal dimension of a positive-definite subspace for the intersection form on $H^{2}(Z, \partial Z ; \mathbb{R})$. It is suggestive to compare the hypothesis that $|n| \geq 2 g-1$ with the "adjunction inequality" for surfaces of non-negative square (see [7] or [13]). Indeed, the hypotheses of Theorem 1.1 are sharp: if we allow $b_{2}^{+}\left(X_{2}\right)=0$ or $|n| \leq 2 g-2$, there are many examples of such splittings, obtained by blowing up smoothly embedded complex curves $C$ in $X$, and splitting $X$ along the boundary of the tubular neighborhood of $C$.

Moreover, the situation for elliptic surfaces is quite different, as we see below:

Theorem 1.2. (1) Every simply-connected elliptic surface with $b_{2}^{+}(X)>3$ admits a splitting along $Y(1,1)$ with $b_{2}^{+}\left(X_{i}\right)>0$.

(2) For each $n>0$, there is a simply-connected elliptic surface $X$ which admits a splitting along $Y(n, 1)$ with $b_{2}^{+}\left(X_{i}\right)>0$.

Proof. A splitting of Type (1) is given as follows. Note that $Y(1,1)$ is the mapping cylinder of a (single) Dehn twist on the torus. Thus, if we begin with the rational elliptic surface

$$
\pi: E(1)=\mathbb{C P}^{2} \# 9 \overline{\mathbb{C P}}^{2} \longrightarrow \mathbb{C P}^{1},
$$

Received December 22, 1999.

The first author was partially supported by NSF grant number DMS 9971950.

The second author was partially supported by NSF grant number DMS 970435 and a Sloan Fellowship. 
let $x \in \mathbb{C P}^{1}$ denote the image of a fishtail fiber, and let $\Delta$ be a disk around $x$ containing no other singular points for $\pi$, then $\pi^{-1}(\partial \Delta)=Y(1,1)$ splits $E(1)$ into a pair of elliptic fibrations $Z_{1}$ and $Z_{2}$ over disks. Thus we can realize $E(3)$ as a union of fiber sums $E(1) \# Z_{1}$ and $Z_{2} \# E(1)$ joined along $Y(1,1)$, where $E(3)$ is the fiber sum of three copies of $E(1)$. Neither side is negative-definite: both sides contain a torus of square zero and a sphere (constructed from vanishing cycles) which meets this torus in a single, positive point. Since every simply-connected elliptic surface with $b_{2}^{+} \geq 3$ can be obtained from $E(3)$ by fiber sums with $E(1)$, logarithmic transformations, and blow-ups (see [11], or [6]) the result follows.

A splitting of Type (2) is realized by finding an elliptic surface $Z$ over $\mathbb{C P}^{1}$ which contains $n$ singular values for the elliptic fibration whose holonomy is a Dehn twist along a given curve in the fiber. In fact, it is a theorem of Moishezon (see [11], also Theorem 3.6 in Chapter 2 of [6]) that if $Z$ is a nodal elliptic surface without multiple fibers and $2 m$ singular fibers, then we can think of the monodromy representation around $m$ of the singular fibers, of which we select $n$, as being a $(+1)$ Dehn twist around a fixed non-separating curve in the fiber, and the monodromy around the remaining $m$ as being a Dehn twist around another curve. Let $\Delta$ be a disk in $\mathbb{C P}^{1}$ which contains only the $n$ distinguished singular points and no others. Now, it is easy to see that $\pi^{-1}(\partial \Delta)=Y(n, 1)$, which separates the elliptic surface. Forming fiber sums with rational elliptic surfaces on both sides as before, we get a decomposition of the elliptic surface $E(1) \# Z \# E(1)$ along $Y(n, 1)$ into two pieces with $b_{2}^{+}>0$.

Remark 1.3. Note that the hypothesis that $b_{2}^{+}(Z)>3$ above is necessary: the elliptic surface $E(2)$ admits no decomposition along $Y(n, g)$ with $g \geq 2 n-1$ and $b_{2}^{+}\left(X_{i}\right)>0$. This follows from the fact that $E(2)$ has a single basic class, together with the vanishing result, Theorem 2.1, from Section 2.

Remark 1.4. Using the above decomposition (Type 2) as a building block, it is possible to construct symplectic four-manifolds $Z$ which decompose along $Y(n, g)$ with $n$ and $g$ arbitrarily large, such that both sides have positive $b_{2}^{+}$. For example, one can start with an elliptic surface $X$ decomposed along $Y(n, 1)$ in the manner of Theorem 1.2, and find a symplectic torus $T \subset X$ (which is symplectic for a form arbitrarily close to a Kähler form for $X$ ) which meets $Y(n, 1)$ in a fiber circle for the Seifert fibration of $Y(n, 1)$, and has square zero. Forming the fiber sum of $X$ with, say, $T^{2} \times \Sigma_{g-1}$ (by gluing $T \subset X$ to $T^{2} \times p$ ), we obtain $Z$ as claimed.

Theorem 1.1 follows from a "vanishing theorem," Theorem 2.1, according to which a certain sum of Seiberg-Witten invariants for $X$ vanishes whenever $X$ splits into two pieces with $b_{2}^{+}\left(X_{i}\right)>0$ along $Y(n, g)$, when $|n| \geq 2 g-1$. This is a more refined vanishing statement than the usual vanishing theorem for manifolds which split along $S^{3}$; in particular there are manifolds with nontrivial Seiberg-Witten invariants which satisfy the hypotheses of Theorem 1.1, as is illustrated by Theorem 1.2. The vanishing theorem (Theorem 2.1) is proved by looking at the ends of the moduli spaces of flows to the reducibles: this is also 
the philosophy adopted by Austin and Braam in [1], see also [16]. In the case where $g=1$, it is interesting to compare the vanishing theorem with a certain vanishing theorem for Donaldson polynomials proved by Morgan, Mrowka, and Ruberman (Theorem 16.0.1 of [12]).

We will give the proof of Theorem 1.1 in Section 2, after stating and proving the more general vanishing result on which it is based.

\section{The Vanishing Theorem}

To state the vanishing theorem which implies Theorem 1.1, we must introduce some notation. We think of the Seiberg-Witten invariant of a smooth, oriented, closed four-manifold $X$ (with a "homology orientation" - an orientation on $\left(H^{0} \oplus\right.$ $\left.\left.H^{1} \oplus H^{+}\right)(X ; \mathbb{R})\right)$ and $\operatorname{Spin}^{c}$ structure $\mathfrak{s}$ as a homogenous polynomial map

$$
S W_{(X, \mathfrak{s})}: \mathbb{A}(X) \longrightarrow \mathbb{Z}
$$

of degree

$$
d(\mathfrak{s})=\frac{c_{1}(\mathfrak{s})^{2}-(2 \chi+3 \sigma)}{4}
$$

on the algebra

$$
\mathbb{A}(X)=\mathbb{Z}[U] \otimes_{\mathbb{Z}} \Lambda^{*} H_{1}(X ; \mathbb{Z}),
$$

where $U$ is a two-dimensional generator, and $\Lambda^{*} H_{1}(X ; \mathbb{Z})$ is the exterior algebra on the first homology of $X$ (graded in the obvious manner). This algebra maps surjectively to the cohomology ring of the irreducible configuration space $\mathcal{B}^{*}(X, \mathfrak{s})$ of pairs $[A, \Phi]$ of $\operatorname{Spin}^{c}$ connections $A$ and somewhere non-vanishing spinors $\Phi$ modulo gauge. (We denote the full configuration space of pairs modulo gauge, i.e. where $\Phi$ is allowed to vanish, by $\mathcal{B}(X, \mathfrak{s})$.) As usual, the SeibergWitten invariant is obtained by cohomological pairings of these cohomology classes with the fundamental cycle of the moduli space $\mathcal{M}(X, \mathfrak{s})$ of solutions to the Seiberg-Witten equations, which is naturally induced from the homology orientation.

As in Section 1, let $Y=Y(n, g)$ be the circle bundle over a Riemann surface with Euler number $n$ over a surface $\Sigma$ of genus $g>0$. Throughout this section, we assume that

$$
|n| \geq 2 g-1 \text {. }
$$

Recall that $H^{2}(Y ; \mathbb{Z}) \cong \mathbb{Z}^{2 g} \oplus(\mathbb{Z} / n \mathbb{Z})$, where the $\mathbb{Z} / n \mathbb{Z}$ factor is generated by multiples of the pull-back $\pi^{*}$ of the orientation class of $\Sigma$. Indeed, there is a canonical $\operatorname{Spin}^{c}$ structure $\mathfrak{t}_{0}$ over $Y$ associated to the two-plane field orthogonal to the circle directions. Thus, forming the tensor product with $\mathfrak{t}_{0}$ gives a canonical identification

$$
\operatorname{Spin}^{c}(Y) \cong H^{2}(Y ; \mathbb{Z}) .
$$

In particular, there are $n$ distinguished $\operatorname{Spin}^{c}$ structures $\mathfrak{t}_{e}$ over $Y$, indexed by $e \in \mathbb{Z} / n \mathbb{Z}$ (thought of as a subset of $H^{2}(Y ; \mathbb{Z})$ ). 
Theorem 2.1. Let $X$ be a smooth, closed, oriented four-manifold which splits along an embedded copy of $Y=Y(n, g)$ with $|n|>2 g-1$, so that $X=X_{1} \#_{Y} X_{2}$ with $b_{2}^{+}\left(X_{i}\right)>0$ for $i=1,2$. Fix a Spin ${ }^{c}$ structure $\mathfrak{s}$ on $X$, and let $\left.\mathfrak{s}\right|_{Y}=\mathfrak{t}$. If $\mathfrak{t}$ is not one of the $n$ distinguished $\operatorname{Spin}^{c}$ structures on $Y$, then $S W_{(X, \mathfrak{s})} \equiv 0$. Similarly, if $\mathfrak{t}=\mathfrak{t}_{e}$ for $2 g-2<e<n$, then $S W_{(X, \mathfrak{s})} \equiv 0$. Otherwise, if $\mathfrak{t}=\mathfrak{t}_{e}$ for $i=0, \ldots, 2 g-2$, we have that

$$
\sum_{\left\{\mathfrak{s}^{\prime}\left|\mathfrak{s}^{\prime}\right|_{X_{1}}=\left.\mathfrak{s}\right|_{\left.X_{1},\left.\mathfrak{s}^{\prime}\right|_{X_{2}}=\left.\mathfrak{s}\right|_{X_{2}}\right\}}\right.} S W_{\left(X, \mathfrak{s}^{\prime}\right)} \equiv 0
$$

Note that the inclusion $Y \subset X$ gives rise to a coboundary homomorphism $\delta: H^{1}(Y ; \mathbb{Z}) \rightarrow H^{2}(Y ; \mathbb{Z})$, whose image we denote by $\delta H^{1}(Y ; \mathbb{Z})$. Another way of stating Equation (1) is:

$$
\sum_{\eta \in \delta H^{1}(Y ; \mathbb{Z})} S W_{(X, \mathfrak{s}+\eta)} \equiv 0 .
$$

The above theorem is proved by considering the ends of certain moduli spaces over cylindrical-end manifolds. In general, these ends are described in terms of the moduli spaces of the boundary $Y$, and the moduli spaces of solutions on the cylinder $\mathbb{R} \times Y$ (using a product metric and perturbation).

Specifically, let $Y$ be a three-manifold, and let $\mathcal{N}_{Y}(\mathfrak{t})$ denote the moduli space of solutions to the three-dimensional Seiberg-Witten equations over $Y$ in the Spin $^{c}$ structure $\mathfrak{t}$. Given a pair of components $C_{1}, C_{2}$ in $\mathcal{N}_{Y}(\mathfrak{t})$, let $\mathcal{M}\left(C_{1}, C_{2}\right)$ denote the moduli space of solutions $[A, \Phi]$ to the Seiberg-Witten equations on $\mathbb{R} \times Y$ for which

$$
\left.\lim _{t \mapsto-\infty}[A, \Phi]\right|_{\{t\} \times Y} \in C_{1} \quad \text { and }\left.\quad \lim _{t \mapsto \infty}[A, \Phi]\right|_{\{t\} \times Y} \in C_{2}
$$

The theory of [12] can be adapted to give the moduli space $\mathcal{M}\left(C_{1}, C_{2}\right)$ a Fredholm deformation theory, and a pair of continuous "boundary value maps" for $i=1,2$

characterized by

$$
\rho_{C_{i}}: \mathcal{M}\left(C_{1}, C_{2}\right) \longrightarrow C_{i}
$$

$$
\rho_{C_{1}}[A, \Phi]=\left.\lim _{t \mapsto-\infty}[A, \Phi]\right|_{\{t\} \times Y} \quad \text { and } \quad \rho_{C_{2}}[A, \Phi]=\left.\lim _{t \mapsto+\infty}[A, \Phi]\right|_{\{t\} \times Y} .
$$

The moduli space $\mathcal{M}\left(C_{1}, C_{2}\right)$ admits a translation action by $\mathbb{R}$, and we let $\widehat{\mathcal{M}}\left(C_{1}, C_{2}\right)$ denote the quotient of this space by this action. The boundary value maps are $\mathbb{R}$-invariant, and hence induce boundary value maps on the quotient

$$
\rho_{C_{i}},: \widehat{\mathcal{M}}\left(C_{1}, C_{2}\right) \longrightarrow C_{i} \text {. }
$$

As in [7] (by analogy with the cases considered by Floer, see for instance [4]), the solutions to the three-dimensional Seiberg-Witten equations are the critical points for a "Chern-Simons-Dirac" functional CSD defined on the configuration space $\mathcal{B}(Y, \mathfrak{t})$. The Seiberg-Witten equations on $\mathbb{R} \times Y$ can be naturally identified with upward gradient flowlines for this functional. (Strictly speaking, the 
functional CSD is real-valued only when the first Chern class $c_{1}(\mathfrak{t})$ is torsion; otherwise it is circle-valued.)

Solutions in $\mathcal{N}(Y, \mathfrak{t})$ whose spinor vanishes identically correspond to flat connections on the determinant line bundle for $\mathfrak{t}$. By analogy with the DonaldsonFloer theory, these solutions are usually called reducibles, and those with somewhere non-vanishing spinor are called irreducibles.

In the case where $Y$ is a non-trivial circle bundle over a Riemann surface these moduli spaces were studied in [14], see also [15] (where $Y$ is endowed with a circle-invariant metric and the Seiberg-Witten equations over it are suitably perturbed). Specifically, there is the following result:

Theorem 2.2. Let $Y$ be a circle bundle over a Riemann surface with genus $g>0$, and Euler number $|n|>2 g-2$ (oriented as circle bundle with negative Euler number). Then, the moduli space $\mathcal{N}_{Y}(\mathfrak{t})$ is empty unless $\mathfrak{t}$ corresponds to a torsion class in $H^{2}(Y ; \mathbb{Z})$. Suppose that $\mathfrak{t}=\mathfrak{t}_{e}$ for $e \in \mathbb{Z} / n \mathbb{Z} \subset H^{2}(Y ; \mathbb{Z})$. Then

(1) If $0 \leq e \leq g-1$ then $\mathcal{N}_{Y}(\mathfrak{t})$ contains two components, a reducible one $\mathcal{J}$, identified with the Jacobian torus $H^{1}\left(\Sigma ; S^{1}\right)$, and a smooth irreducible component $C$ diffeomorphic to $\operatorname{Sym}^{e}(\Sigma)$. Both of these components are non-degenerate in the sense of Morse-Bott. There is an inequality $\operatorname{CSD}(\mathcal{J})>\operatorname{CSD}(C)$, so the space $\widehat{\mathcal{M}}(\mathcal{J}, C)$ is empty. The space $\widehat{\mathcal{M}}(C, \mathcal{J})$ is smooth of expected dimension $2 e$; indeed it is diffeomorphic to $\operatorname{Sym}^{e}(\Sigma)$ under the boundary value map

$$
\rho_{C}: \widehat{\mathcal{M}}(C, \mathcal{J}) \longrightarrow C \cong \operatorname{Sym}^{e}(\Sigma)
$$

(2) If $g-1<e \leq 2 g-2$, the Seiberg-Witten moduli spaces over both $Y$ and $\mathbb{R} \times Y$ in this Spin $^{c}$ structure are naturally identified with the corresponding moduli spaces in the $\operatorname{Spin}^{c}$ structure $2 g-2-e$, which we just described.

(3) For all other e, $\mathcal{N}_{Y}(\mathfrak{t})$ contains only reducibles. Furthermore, it is smoothly identified with the Jacobian torus $\mathcal{J}$.

When $e \neq g-1$, the above theorem is a special case of Theorems 1 and 2 of [14] (see especially Corollary 1.5 of [14]). When $e=g-1$, the case considered in that paper is not "generic". In fact, there is a natural perturbation (by some small multiple of the connection 1-form of the Seifert fibration), which achieves the genericity stated above. This perturbation was used in [15] to prove strong "adjunction inequalities" for manifolds which are not of simple type, and the above theorem in the case where $e=g-1$ is precisely Theorem 8.1 of [15]. Note that the hypothesis $n>2 g-2$ is required to separate the irreducible manifolds into distinct $\operatorname{Spin}^{c}$ structures. Note also that if the orientation on $Y$ is reversed, the flow-lines reverse direction.

The proof of Theorem 2.1 is obtained by considering the ends of the moduli spaces $\mathcal{M}\left(X_{1}, \mathfrak{s}_{1}, \mathcal{J}\right)$ of Seiberg-Witten monopoles over the cylindrical-end 
manifold

$$
X_{1}^{+}=X_{1} \cup_{\partial X_{1}=\{0\} \times Y}[0, \infty) \times Y
$$

in the $\operatorname{Spin}^{c}$ structure $\mathfrak{s}_{1}$, whose boundary values are reducible. We will assume, as in that theorem, that $b_{2}^{+}\left(X_{1}\right)>0$. In general, moduli spaces of finite energy solutions to the Seiberg-Witten equations on a manifold with cylindrical ends are not compact. (The "finite energy condition" in this context is equivalent to the hypothesis that the pair $[A, \Phi]$ has a well-defined boundary value.) They do, however, have "broken flowline" compactifications (see [12] and [4]). In particular, if $C$ is a component of $\mathcal{N}\left(Y,\left.\mathfrak{s}_{1}\right|_{Y}\right)$, then for generic perturbations, the moduli space $\mathcal{M}\left(X_{1}, \mathfrak{s}_{1}, C\right)$ is a smooth manifold with finitely many ends indexed by components $C_{1}, \ldots, C_{n}$ in the moduli space $\mathcal{N}\left(Y,\left.\mathfrak{s}_{1}\right|_{Y}\right)$, with $\operatorname{CSD}\left(C_{1}\right)<$ $\operatorname{CSD}\left(C_{2}\right)<\ldots<\operatorname{CSD}\left(C_{n}\right)<\operatorname{CSD}(C)$. When all the $C_{i}$ are non-degenerate in the sense of Morse-Bott, and consist of irreducibles, a neighborhood of the corresponding end is diffeomorphic to the fibered product

$$
\mathcal{M}\left(X_{1}, \mathfrak{s}_{1}, C_{1}\right) \times_{C_{1}} \mathcal{M}\left(C_{1}, C_{2}\right) \times_{C_{2}} \ldots \times_{C_{n}} \mathcal{M}\left(C_{n}, C\right),
$$

under a certain gluing map (provided that this space is a manifold - i.e. provided that the various boundary value maps are transverse).

In particular, suppose $X_{1}$ is an oriented four-manifold with boundary, whose boundary $\partial X_{1}$ is identified with $Y=Y(n, g)$ with the orientation described in Theorem 2.2. Then, it follows from that theorem that if $\left.\mathfrak{s}_{1}\right|_{Y}=\mathfrak{t}_{e}$ for $0 \leq e \leq$ $2 g-2$, then $\mathcal{M}\left(X_{1}, \mathfrak{s}_{1}, C\right)$ is compact (since there are no "intermediate" critical manifolds to be added), and $\mathcal{M}\left(X_{1}, \mathfrak{s}_{1}, \mathcal{J}\right)$ has a single end whose neighborhood is diffeomorphic to

$$
\mathcal{M}\left(X_{1}, \mathfrak{s}_{1}, C\right) \times(0, \infty) .
$$

(We use here the fact that the restriction map $\rho_{C}: \widehat{\mathcal{M}}(C, \mathcal{J}) \longrightarrow C$ is a diffeomorphism.) This gluing map

$$
\gamma: \mathcal{M}\left(X_{1}, \mathfrak{s}_{1}, C\right) \times(0, \infty) \longrightarrow \mathcal{M}\left(X_{1}, \mathcal{J}\right)
$$

is compatible with restriction to compact subsets of $X_{1}^{+}$; e.g. if we consider the compact subset $X_{1} \subset X_{1}^{+}$, then

$$
\left.\lim _{T \mapsto \infty} \gamma([A, \Phi], T)\right|_{X_{1}}=\left.[A, \Phi]\right|_{X_{1}} .
$$

We make use of the end of $\mathcal{M}\left(X_{1}, \mathfrak{s}_{1}, \mathcal{J}\right)$ in the following proposition. Recall that the moduli space $\mathcal{M}\left(X_{1}, \mathfrak{s}_{1}, C\right)$ is a smooth, compact submanifold of the irreducible configuration space of $X_{1}^{+}$. It has a canonical top-dimensional homology class, denoted $\left[\mathcal{M}\left(X_{1}, \mathfrak{s}_{1}, C\right)\right]$, induced from the "homology orientation" of $X_{1}$. It inherits cohomology classes by pulling back via the boundary value map

$$
\rho_{C}: \mathcal{M}\left(X_{1}, \mathfrak{s}_{1}, C\right)
$$

and from the natural map

$$
i_{X_{1}}: \mathcal{M}\left(X_{1}, \mathfrak{s}\right) \longrightarrow \mathcal{B}^{*}\left(X_{1}, \mathfrak{s}\right)
$$


given by restricting the pair $[A, \Phi]$ to the compact subset $X_{1} \subset X_{1}^{+}$(this restriction is irreducible according to the unique continuation theorem for the Dirac operator). The pairings with these classes can be thought of as a "relative Seiberg-Witten" invariant.

Proposition 2.3. Suppose $b_{2}^{+}\left(X_{1}\right)>0$. Given any cohomology classes a $\in$ $H^{*}\left(\mathcal{B}^{*}\left(X_{1}, \mathfrak{s}_{1}\right)\right)$ and $b \in H^{*}(C)$, the homology class $\left[\mathcal{M}\left(X_{1}, \mathfrak{s}_{1}, C\right)\right]$ pairs trivially with the class $i_{X_{1}}^{*}(a) \cup \rho_{C}^{*}(b)$.

Proof. First, we reduce to the case where $b$ is absent (i.e. zero-dimensional). This is done in two steps, first establishing an inclusion

$$
\left(i_{Y} \circ \rho_{C}\right)^{*} H^{*}\left(\mathcal{B}^{*}(Y, \mathfrak{t})\right) \subset i_{X_{1}}^{*}\left(\mathcal{B}^{*}\left(X_{1}, \mathfrak{s}_{1}\right)\right),
$$

where both are thought of as subsets of $H^{*}\left(\mathcal{M}\left(X_{1}, \mathfrak{s}_{1}, C\right)\right)$, and then seeing that the map

is surjective.

$$
i_{Y}^{*}: H^{*}\left(\mathcal{B}^{*}(Y, \mathfrak{t})\right) \longrightarrow H^{*}(C)
$$

To see Inclusion (2) we describe the geometric representatives for the generators of the cohomology ring

$$
H^{*}\left(\mathcal{B}^{*}(Y, \mathfrak{t})\right) \cong \mathbb{Z}[U] \otimes_{\mathbb{Z}} \Lambda^{*}\left(H_{1}(Y ; \mathbb{Z})\right)
$$

Given a point $y \in Y$ and a line $\Lambda_{y} \subset W_{y}$ in the fiber of the spinor bundle over $y$, the class $U$ is Poincaré dual to the locus $V_{\left(y, \Lambda_{y}\right)}$ of pairs $[B, \Psi]$ with $\Psi_{y} \in$ $\Lambda_{y}$. Moreover, given a curve $\gamma: S^{1} \rightarrow X$, the corresponding one-dimensional cohomology class determined by the homotopy type of the map

$$
h_{\gamma}: \mathcal{B}^{*}(Y, \mathfrak{t}) \longrightarrow S^{1}
$$

given by measuring the holonomy of $B$ (relative to some fixed reference connection $B_{0}$ ) around $\gamma$; i.e. it is Poincaré dual to the preimage $V_{\gamma}$ of a regular value of $h_{\gamma}$. This cohomology class is denoted $\mu[\gamma] \in H^{1}\left(\mathcal{B}^{*}(Y, \mathfrak{t})\right.$ ). (Note that geometric representatives cohomology classes in the configuration spaces of four-manifolds are constructed in an analogous manner.)

Now, fix a curve $\gamma \subset Y$ and consider the one-parameter family of maps

$$
h_{t}: \mathcal{M}\left(X_{1}, \mathfrak{s}_{1}, C\right) \longrightarrow S^{1}
$$

indexed by $t \in(0,1]$ defined by measuring the holonomy of $A$ around the curve $\{1 / t\} \times \gamma \subset X_{1}^{+}$. Since the configurations in $[A, \Phi] \in \mathcal{M}\left(X_{1}, \mathfrak{s}_{1}, C\right)$ converge exponentially to a stationary solution (see [12]), $h_{t}$ extends continuously to $t=0$. Now, $h_{0}$ represents $\left(i_{Y} \circ \rho_{C}\right) *$ of the one-dimensional class $\mu[\gamma] \in H^{1}\left(\mathcal{B}^{*}(Y, \mathfrak{t})\right)$, while $h_{1}$ represents the restriction (to the moduli space) of the one-dimensional class $\mu\left[\gamma_{1}\right] \in H^{*}\left(\mathcal{B}^{*}\left(X_{1}, \mathfrak{s}_{1}\right)\right)$, where $\mu_{1}=\{1\} \times Y \subset X_{1}$. A similar discusion applies to the two-dimensional class to show that $\rho_{C}^{*} U=i_{X_{1}}^{*} U$ (now we use the connection $A$ to identify the fiber "at infinity" with the fiber at some point inside $X$ ). This completes the verification of Inclusion (2).

Surjectivity of

$$
i_{Y}^{*}: H^{*}\left(\mathcal{B}^{*}(Y, \mathfrak{t}) ; \mathbb{Z}\right) \cong \mathbb{A}(Y) \rightarrow H^{*}(C ; \mathbb{Z})
$$


follows from classical properties of the cohomology of symmetric products $\operatorname{Sym}^{e}(\Sigma)$ (see [9]), according to which the cohomology ring is generated by "symmetrizations" of the cohomology of $\Sigma$. It is then a straightforward verification (which is spelled out in Proposition 6.10 of [15]) using the geometric interpretations of the cohomology classes given above to see that that $i_{Y}^{*} \mu(\gamma)$ corresponds to the symmetrization of $\pi(\gamma)$, while $i_{Y}^{*} U$ corresponds to the symmetrization of the point $\pi(x)$ on $\Sigma$ (where we think of $U$ as the Poincaré dual of $V_{(x, \Lambda)}$ for some choice of line $\Lambda \subset W_{x}$ ).

Thus, it remains to prove that $\left[\mathcal{M}\left(X_{1}, \mathfrak{s}_{1}, C\right)\right]$ pairs trivially with classes in

$$
i_{X_{1}}^{*} H^{*}\left(\mathcal{B}^{*}\left(X_{1}, \mathfrak{s}_{1}\right)\right) .
$$

We can think of the cohomological pairing $\left\langle\rho_{C}^{*}(b),\left[\mathcal{M}\left(X_{1}, \mathfrak{s}_{1}, C\right)\right]\right\rangle$ as counting the (signed) number of points to the Seiberg-Witten equations, which satisfy constraints in the compact subset $X_{1} \subset X_{1}^{+}$; i.e. if $b=U^{d} \cdot\left[\mu_{1}\right] \cdot \ldots \cdot\left[\mu_{\ell}\right]$, where $\mu_{i}$ are curves in $X_{1}$, and $x_{1}, \ldots, x_{d}$ are generic points in $X_{1}$, and $\Lambda_{1}, \ldots, \Lambda_{d}$ are generic lines $\left.\Lambda_{i} \subset W^{+}\right|_{\left\{x_{i}\right\}}$, then we have gemetric representatives $V_{\left(x_{i}, \Lambda_{i}\right)}$ and $V_{\mu_{i}}$ for these cohomology classes, so that

$$
\left\langle\left[\mathcal{M}\left(X_{1}, \mathfrak{s}_{1}\right)\right], b\right\rangle=\# \mathcal{M}\left(X_{1}\right) \cap V
$$

where $V=V_{\left(x_{1}, \Lambda_{1}\right)} \cap \ldots \cap V_{\left(x_{d}, \Lambda_{d}\right)} \cap V_{\mu_{1}} \cap \ldots \cap V_{\mu_{\ell}}$. In fact, if we consider the solutions $\mathcal{M}\left(X_{1}, \mathfrak{s}_{1}, \mathcal{J}\right)$ which satisfy these same constraints, then we get a manifold of dimension one with ends corresponding to $\mathcal{M}\left(X_{1}, \mathfrak{s}_{1}, C\right) \cap V$. Thus, counting boundary points with sign, we see that

$$
\# \mathcal{M}\left(X_{1}, \mathfrak{s}_{1}, C\right) \cap V=0 .
$$

Proof of Theorem 2.1. In the splitting $X=X_{1} \cup_{Y} X_{2}$, we can number the sides so that the boundary of $X_{1}$ is $Y$ oriented as in Theorem 2.2. Let $\mathfrak{s}$ be a $\operatorname{Spin}^{c}$ structure over $X$, and let $\mathfrak{s}_{i} \in \operatorname{Spin}^{c}\left(X_{i}\right)$ denote the restriction $\mathfrak{s}_{i}=\left.\mathfrak{s}\right|_{X_{i}}$ for $i=1,2$, and let $\mathfrak{t} \in \operatorname{Spin}^{c}(Y)$ denote the restriction $\mathfrak{t}=\left.\mathfrak{s}\right|_{Y}$. Let $X(T)$ denote the Riemannian structure on $X$ obtained by inserting a cylinder $[-T, T] \times Y$ between $X_{1}$ and $X_{2}$ (but keeping the metrics on these two pieces to be fixed, and product-like near the boundary). If the Seiberg-Witten invariants for a Spin $^{c}$ structure $\mathfrak{s}$ over $X$ is non-trivial, for any unbounded, increasing sequence of real numbers $\left\{T_{i}\right\}$, there must be a sequence of Seiberg-Witten monopoles $\left[A_{i}, \Phi_{i}\right] \in \mathcal{M}\left(X\left(T_{i}\right), \mathfrak{s}\right)$. The uniform bound in the energy, and local compactness (see [7]) allows one to find a sequence $\left\{t_{i}\right\}$ with $t_{i} \leq T_{i}$, so that, after passing to a subsequence if necessary, $\left.\left[A_{i}, \Phi_{i}\right]\right|_{\left\{t_{i}\right\} \times Y}$ converges to a stationary solution; i.e. it converges to a point in $\mathcal{N}(Y, \mathfrak{t})$. Thus, from Theorem 2.2, it follows that the Seiberg-Witten invariant for $\mathfrak{s}$ vanishes unless $\mathfrak{t}$ is one of the $n$ distinguished $\operatorname{Spin}^{c}$ structures $\mathfrak{t}_{e}$ over $Y$.

Suppose that $\mathfrak{t}=\mathfrak{t}_{e}$, for $e=0, \ldots, 2 g$. Note the excision principle for the index gives that

$$
d(\mathfrak{s})=\operatorname{dim} \mathcal{M}\left(X_{1}, \mathfrak{s}_{1}, C\right)+\operatorname{dim} \mathcal{M}\left(X_{2}, \mathfrak{s}_{2}, C\right)-\operatorname{dim}(C)
$$


for any $\operatorname{Spin}^{c}$ structure $\mathfrak{s} \in \operatorname{Spin}^{c}(X)$ with $\left.\mathfrak{s}\right|_{X_{i}}=\mathfrak{s}_{i}$ for $i=1,2$ (and generic, compactly supported perturbations of the equations over $X_{i}$ ). We fix an integer $\ell \geq 0$ and homology classes $a_{1}, \ldots, a_{m} \in H_{1}\left(X_{1} ; \mathbb{Z}\right), b_{1}, \ldots, b_{n} \in H_{1}\left(X_{2} ; \mathbb{Z}\right)$ with

$$
2 \ell+m+n=d(\mathfrak{s}) .
$$

Let $\mathfrak{s}_{1} \# \mathfrak{s}_{2} \subset \operatorname{Spin}^{c}(X)$ denote the subset of $\operatorname{Spin}^{c}$ structures on $X$ :

$$
\mathfrak{s}_{1} \# \mathfrak{s}_{2}=\left\{\mathfrak{s}^{\prime} \in \operatorname{Spin}^{c}(X)\left|\mathfrak{s}^{\prime}\right|_{X_{1}}=\mathfrak{s}_{1},\left.\mathfrak{s}^{\prime}\right|_{X_{2}}=\mathfrak{s}_{2}\right\},
$$

and let $\mathcal{M}\left(X(T), \mathfrak{s}_{1} \# \mathfrak{s}_{2}\right)$ denote the union

$$
\mathcal{M}\left(X(T), \mathfrak{s}_{1} \# \mathfrak{s}_{2}\right)=\coprod_{\mathfrak{s}^{\prime} \in \mathfrak{s}_{1} \# \mathfrak{s}_{2}} \mathcal{M}\left(X, \mathfrak{s}^{\prime}\right)
$$

Clearly, we have that

$$
\# \mathcal{M}\left(X(T), \mathfrak{s}_{1} \# \mathfrak{s}_{2}\right) \cap V_{1} \cap V_{2}=\sum_{\mathfrak{s}^{\prime} \in \mathfrak{s}_{1} \# \mathfrak{s}_{2}} S W_{X, \mathfrak{s}^{\prime}}\left(U^{\ell} \cdot\left[a_{1}\right] \cdot \ldots \cdot\left[a_{m}\right] \cdot\left[b_{1}\right] \cdot \ldots \cdot\left[b_{n}\right]\right)
$$

where $V_{i}$ are the intersection of the constraints from the $X_{i}$ side; e.g.

$$
V_{1}=V_{\left(x_{1}, \Lambda_{1}\right)} \cap \ldots \cap V_{\left(x_{\ell}, \Lambda_{m}\right)} \cap V_{a_{1}} \cap \ldots \cap V_{a_{m}}
$$

and

$$
V_{2}=V_{b_{1}} \cap \ldots \cap V_{b_{n}} .
$$

Thus, our aim is to prove that the total signed number of points in the cut-down moduli space $\mathcal{M}\left(X(T), \mathfrak{s}_{1} \# \mathfrak{s}_{2}\right) \cap V_{1} \cap V_{2}$ is zero.

Given pre-compact sets $K_{i} \subset \mathcal{M}\left(X_{i}, \mathfrak{s}_{i}, C\right)$ for $i=1,2$, there are gluing maps defined for all sufficiently large $T$,

$$
\gamma_{C ; T}: K_{1} \#_{C} K_{2} \longrightarrow \mathcal{M}\left(X(T), \mathfrak{s}_{1} \# \mathfrak{s}_{2}\right),
$$

where the domain is the fibered product of $K_{1}$ and $K_{2}$ over $\rho_{1}$ and $\rho_{2}$, i.e. the set of $\left[A_{1}, \Phi_{1}\right] \in K_{1},\left[A_{2}, \Phi_{2}\right] \in K_{2}$ with

$$
\rho_{1}\left(\left[A_{1}, \Phi_{1}\right]\right)=\rho_{2}\left(\left[A_{2}, \Phi_{2}\right]\right),
$$

and the range consists of all configurations $[A, \Phi]$ whose restrictions to $X_{1}$ and $X_{2}$ are sufficiently close to restrictions (to $X_{1}$ and $X_{2}$ ) of configurations $\left[A_{1}, \Phi\right] \times$ $\left[A_{2}, \Phi_{2}\right]$ in the fibered product.

We claim that for all sufficiently large $T$, the cut-down moduli space lies in the range of this map. Specifically, if we had a sequence $\left[A_{i}, \Phi_{i}\right] \in \mathcal{M}\left(X\left(T_{i}\right), \mathfrak{s}_{1} \# \mathfrak{s}_{2}\right)$ for an increasing, unbounded sequence $\left\{T_{i}\right\}_{i=1}^{\infty}$ of real numbers, the sequence converges $C^{\infty}$ locally to give a pair of Seiberg-Witten monopoles monopoles $\left[A_{1}, \Phi_{1}\right] \in \mathcal{M}\left(X_{1}, \mathfrak{s}_{1}\right)$ and $\left[A_{2}, \Phi_{2}\right] \in \mathcal{M}\left(X_{2}, \mathfrak{s}_{2}\right)$. These monopoles have finite energy (since the total variation of CSD is bounded in the limit), so they have boundary values, which must lie in either $C$ or $\mathcal{J}$. We exclude all but one of the four cases as follows:

(C-1) The case where $\rho_{1}\left[A_{1}, \Phi_{1}\right] \in \mathcal{J}$ and $\rho_{2}\left[A_{2}, \Phi_{2}\right] \in C$ is excluded since $\operatorname{CSD}(C)<\operatorname{CSD}(\mathcal{J})$. 
(C-2) The case where $\rho_{1}\left[A_{1}, \Phi_{1}\right] \in \mathcal{J}$ and $\rho_{2}\left[A_{2}, \Phi_{2}\right] \in \mathcal{J}$ is excluded by a dimension count. Specifically, we must have that

$$
\rho_{1}\left[A_{1}, \Phi_{1}\right]=\rho_{2}\left[A_{2}, \Phi_{2}\right]
$$

and $\left[A_{1}, \Phi_{1}\right] \in \mathcal{M}\left(X_{1}, \mathcal{J}\right) \cap V_{1}$ and $\left[A_{2}, \Phi_{2}\right] \in \mathcal{M}\left(X_{2}, \mathcal{J}\right) \cap V_{2}$, i.e. the pair $\left[A_{1}, \Phi_{1}\right] \times\left[A_{2}, \Phi_{2}\right]$ lies in the fibered product $\mathcal{M}\left(X_{1}, \mathfrak{s}_{1}, \mathcal{J}\right) \times \mathcal{J}$ $\mathcal{M}\left(X_{2}, \mathfrak{s}_{2}, \mathcal{J}\right)$, a space whose dimension is one less than the expected dimension $d(\mathfrak{s})$ of the moduli space. Thus, for generic representatives $V_{1}$ and $V_{2}$, this intersection is empty.

(C-3) The case where $\rho_{1}\left[A_{1}, \Phi_{1}\right] \in C$ and $\rho_{2}\left[A_{2}, \Phi_{2}\right] \in \mathcal{J}$ is excluded by a similar dimension count. We have that $\left[A_{1}, \Phi_{1}\right] \in \mathcal{M}\left(X_{1}, \mathfrak{s}_{1}, C\right) \cap$ $V_{1},\left[A_{2}, \Phi_{2}\right] \in \mathcal{M}\left(X_{2}, \mathfrak{s}_{2}, \mathcal{J}\right) \cap V_{2}$, and $\rho_{1}\left[A_{1}, \Phi_{1}\right]$ is connected to $\rho_{2}\left[A_{2}, \Phi_{2}\right]$ by a (uniquely determined) flow in $\widehat{\mathcal{M}}(C, \mathcal{J})$. This set has expected dimension -2 .

The remaining case is that $\left[A_{1}, \Phi_{1}\right] \in \mathcal{M}\left(X_{1}, \mathfrak{s}_{1}, C\right)$, and $\left[A_{2}, \Phi_{2}\right] \in \mathcal{M}\left(X_{2}, \mathfrak{s}_{2}, C\right)$, with

$$
\rho_{1}\left[A_{1}, \Phi_{1}\right]=\rho_{2}\left[A_{2}, \Phi_{2}\right] .
$$

In particular, $\left[A_{1}, \Phi_{1}\right]$ lies in the compact set $\mathcal{M}\left(X_{1}, \mathfrak{s}_{1}, C\right)$, while $\left[A_{2}, \Phi_{2}\right]$ lies in the set $\rho_{2}^{-1}\left(\rho_{1} \mathcal{M}\left(X_{1}, \mathfrak{s}_{1}, C\right) \cap V_{1}\right) \cap V_{2}$ which is also compact (according to the dimension count used to exclude Case (C-3) above). Thus, for all sufficiently large $T$, the cut-down moduli space lies in the image of the gluing map $\gamma_{C ; T}$.

On compact subsets of $X(T)$ away from the "neck", gluing is a $C^{\infty}$ small perturbation, which goes to zero as the neck-length is increased; in particular, for $i=1,2$,

$$
\left.\lim _{T \mapsto \infty} \gamma_{C ; T}\left(\left[A_{1}, \Phi_{1}\right] \#\left[A_{2}, \Phi_{2}\right]\right)\right|_{X_{i}}=\left.\left[A_{i}, \Phi_{i}\right]\right|_{X_{i}} .
$$

It follows from this that

$$
\begin{aligned}
\# \mathcal{M}\left(X(T), \mathfrak{s}_{1} \# \mathfrak{s}_{2}\right) \cap V_{1} & \cap V_{2}= \\
\# & \left(\left(\mathcal{M}\left(X_{1}, \mathfrak{s}_{1}, C\right) \cap V_{1}\right) \times_{C}\left(\mathcal{M}\left(X_{2}, \mathfrak{s}_{2}, C\right) \cap V_{2}\right)\right) .
\end{aligned}
$$

The latter quantity can be thought of as cohomological pairing in $\mathcal{M}\left(X_{1}, \mathfrak{s}_{1}, C\right)$ as follows.

Fix an oriented, $v$-dimensional submanifold $V \subset \mathcal{M}\left(X_{2}, \mathfrak{s}_{2}, C\right)$, and consider the function which assigns to each smooth map

$$
f: Z \longrightarrow C
$$

(where $Z$ is a smooth, oriented, compact manifold whose dimensionl equals the codimension of $V)$ the number of points in the fibered product $\#\left(Z \times_{C} V\right)$ (counting with sign, after arranging $f$ to be transverse to $V$ ). This is the pairing of the fundamental cycle of $Z$ with an induced cohomology class in $H^{d_{2}-v}(C, \mathbb{Z})$. Indeed, this class can be thought of as the "push-forward" of the Poincaré dual to $V$, under a map

$$
\left(\rho_{2}\right)_{*}: H^{i}\left(\mathcal{M}\left(X_{2}, C\right) ; \mathbb{Z}\right) \longrightarrow H^{i+\operatorname{dim}(C)-d_{2}}(C ; \mathbb{Z}) .
$$


Thus, the count in Equation (3) can be thought of as the pairing

$$
\# \mathcal{M}\left(X(T), \mathfrak{s}_{1} \# \mathfrak{s}_{2}\right) \cap V_{1} \cap V_{2}=\left\langle\left[\mathcal{M}\left(X_{1}, \mathfrak{s}_{1}, C\right)\right], \operatorname{PD}\left(V_{1}\right) \cup \rho_{1}^{*}\left(\rho_{2}\right)_{*} \operatorname{PD}\left(V_{2}\right)\right\rangle .
$$

This pairing vanishes, according to Proposition 2.3. This completes Theorem 2.1 in the case when $\mathfrak{t}=\mathfrak{t}_{e}$ for $e=0, \ldots, 2 g-2$.

In the case when $\mathfrak{t}=\mathfrak{t}_{e}$ for $2 g-1<e<n$, the vanishing of the Seiberg-Witten invariant for any $\mathfrak{s}$ structure with $\left.\mathfrak{s}\right|_{Y}=\mathfrak{t}$ is guaranteed by the same dimension count which we used to exclude Case (C-2) above.

The proof of Theorem 1.1 follows from an application of Theorem 2.1, together with the known properties of Seiberg-Witten invariants for complex surfaces of general type (see for instance [2] or [5], and also [3]), according to which a minimal surface of general type has only two "basic classes" (Spin ${ }^{c}$ structures for which the Seiberg-Witten invariant is non-zero), the "canonical" Spin ${ }^{c}$ structure $\mathfrak{s}_{0}$ (whose first Chern class is given by $c_{1}\left(\mathfrak{s}_{0}\right)=-K_{X}$, where $K_{X} \in H^{2}(X ; \mathbb{Z})$ is the first Chern class of the complex cotangent bundle of $X)$, and its conjugate. Moreover, the basic classes of the $n$-fold blow-up $\widehat{X}=X \# n \overline{\mathbb{C P}}^{2}$ are those Spin ${ }^{c}$ structures $\mathfrak{s}$ whose restriction away from the exceptional spheres agrees with $\mathfrak{s}_{0}$ or its conjugate, and whose first Chern class evaluated on each exceptional sphere $E_{i}$ satisfies

$$
\left\langle c_{1}(\mathfrak{s}),\left[E_{i}\right]\right\rangle= \pm 1
$$

In fact, since $K_{X}^{2}>0$ for a minimal surface of general type, the basic classes are in one-to-one correspondence with their first Chern classes. In view of this fact, throughout the following proof, we label the basic classes of $\widehat{X}$ by their first Chern classes.

Proof of Theorem 1.1. The subgroup $\delta H^{1}(Y ; \mathbb{Z})$ partitions $\operatorname{Spin}^{c}(X)$ into orbits, and Theorem 2.1 states that if $X$ could be decomposed, then the sum of invariants under each orbit vanishes. Note moreover that if $Y$ separates $X$, then the intersection form restricted to the subgroup $\delta H^{1}(Y ; \mathbb{Z})$ is trivial: this is true because we can represent cohomology classes $[\omega],[\eta] \in \delta H^{1}(Y ; \mathbb{R})$ by differential form representatives $\omega$ and $\eta$, with $\left.\omega\right|_{X_{1}} \equiv 0$ and $\left.\eta\right|_{X_{2}} \equiv 0$, so that the representative for $[\omega] \cup[\eta], \omega \wedge \eta$, vanishes identically. It follows from this that in each orbit, there can exist at most two basic classes, for if we had two basic classes which had the same coefficient in $K_{X}$, then their difference would have negative square. Now, suppose that $K_{X}-E_{1}-\ldots-E_{n}$ had another basic class in its orbit. We know that the other basic class would be of the form

$$
-K_{X}+E_{1}+\ldots+E_{a}-E_{a+1}-\ldots-E_{n},
$$

after renumbering the exceptional curves if necessary. The difference $\Delta$ is $2\left(K_{X}-\right.$ $E_{1}-\ldots-E_{a}$ ), which must have square zero, which forces $a>0$ (recall that $K_{X}^{2}>0$ for a minimal surface of general type). Now, consider the basic class $K_{X}+E_{1}+\ldots+E_{n}$. It, too, can have at most one other basic class in its orbit, and the difference has the form

$$
\Delta^{\prime}=2\left(K_{X}+\epsilon_{1} E_{1}+\ldots \epsilon_{n} E_{n}\right),
$$


where we know that $\epsilon_{1}, \ldots, \epsilon_{n} \geq 0$, in particular $\Delta-\Delta^{\prime}$ is a non-zero class, which is easily seen to have negative square. But this contradicts the fact that $\Delta-\Delta^{\prime} \in \delta H^{1}(Y ; \mathbb{Z})$. Thus, it follows that either the basic class $K_{X}-E_{1}-\ldots-E_{n}$ or $K_{X}+E_{1}+\ldots+E_{a}-E_{a+1}-\ldots-E_{n}$ is alone in its $\delta H^{1}(Y ; \mathbb{Z})$ orbit. But this contradicts the conclusion of Theorem 2.1.

2.1. Final remarks. It is suggestive to compare the formal framework adopted here with that of equivariant Morse theory. Specifically, the "ChernSimons-Dirac" operator on $Y$ in the set-up of Theorem 2.2 has precisely two critical manifolds, a manifold of reducibles $\mathcal{J}$ (consisting of configurations whose stabilizer in the gauge group is a circle), and a manifold of irreducibles $C$ (consisting of configurations whose stabilizers are trivial). From the point of view of equivariant cohomology, then, there should be an "equivariant Floer homology", and an analogue of the Bott spectral sequence, whose $E_{2}$ term consists of the homology of the irreducible critical point set $H_{*}\left(\operatorname{Sym}^{e}(\Sigma) ; \mathbb{Z}\right)$, and the $S^{1}$-equivariant homology of the reducible manifold, which is given by

$$
H^{*}\left(\mathbb{C} \mathbb{P}^{\infty} \times \mathcal{J} ; \mathbb{Z}\right) \cong \mathbb{Z}[U] \otimes_{\mathbb{Z}} \Lambda^{*} H_{1}(Y ; \mathbb{Z}) .
$$

From this point of view, Proposition 2.3, upon which the vanishing theorem rests, can be seen then as the calculation of the differential in this spectral sequence.

The equivariant point of view has been stressed by a number of researchers in the field, including (especially in the context of gluing along rational homology three-spheres) [8], [1], [10].

\section{References}

[1] D. M. Austin and P. J. Braam, Morse-Bott Theory and Equivariant Cohomology, (Editors H. Hofer and C. H. Taubes and A. Weinstein and E. Zehnder), The Floer Memorial Volume, Progr. Math. 133 (1995), Birkhäuser, pp. 123-183.

[2] R. Brussee, The canonical class and the $C^{\infty}$ properties of Kähler surfaces, New York J. Math. 2 (1996), 103-146.

[3] R. Fintushel and R. J. Stern, Immersed spheres in 4-manifolds and the immersed Thom conjecture, Turkish J. Math. 19 (1995), 145-157.

[4] A. Floer, An instanton-invariant for 3-manifolds, Comm. Math. Phys. 119 (1988), 215240.

[5] R. Friedman and J. W. Morgan, Algebraic surfaces and Seiberg-Witten invariants, J. Algebraic Geom. 6 (1997), 445-479.

[6] Smooth four-manifolds and complex surfaces, Ergebnisse der Mathematik und ihrer Grenzgebiete (3), Springer-Verlag, 1994.

[7] P. B. Kronheimer and T. S. Mrowka, The genus of embedded surfaces in the projective plane, Math. Res. Lett. 1 (1994), 797-808.

[8] , I.P. Lecture, Irvine, California, 1996.

[9] I. G. MacDonald, Symmetric Products of an Algebraic Curve, Topology 1 (1962), 319343.

[10] M. Marcolli and B.-L. Wang, Equivariant Seiberg-Witten Floer Homology, dg-ga/9606003.

[11] B. Moishezon, Complex surfaces and connected sums of complex projective planes, Lecture Notes in Mathematics 603, Springer-Verlag, 1977. 
[12] J. W. Morgan, T. S. Mrowka, and D. Ruberman, The $L^{2}$-Moduli Space and a Vanishing Theorem for Donaldson Polynomial Invariants, Monographs in Geometry and Topology II, International Press, 1994.

[13] J. W. Morgan, Z. Szabó, and C. H. Taubes, A Product Formula for Seiberg-Witten Invariants and The Generalized Thom Conjecture, J. Differential Geom. 44 (1996), 706-788.

[14] T. S. Mrowka, P. S. Ozsváth, and B. Yu Seiberg-Witten Monopoles on Seifert Fibered Spaces, Comm. Anal. Geom. 5 (1997), 685-791.

[15] P. S. Ozsváth and Z. Szabó, Higher type adjunction inequalities in Seiberg-Witten theory (1998), Preprint.

[16] A. Stipsicz and Z. Szabó Gluing 4-manifolds along $\Sigma(2,3,11)$, Topology Appl. 106 (1999), 293-304.

Department of Mathematics, Princeton University, Princeton, NJ 08540.

E-mail address: petero@math.princeton.edu

Department of Mathematics, Princeton University, Princeton, NJ 08540.

E-mail address: szabo@math.princeton.edu 